\title{
Axillary Reverse Lymphatic Mapping in the Treatment of Axillary Accessory Breast Cancer: A Case Report and Review of Management
}

\author{
(1) Orli Friedman-Eldar ${ }^{1,2}$, (1) Siarhei Melnikau ${ }^{1,2}$, (1) Youley Tjendra ${ }^{3}$, (1) Eli Avisar ${ }^{2}$ \\ ${ }^{1}$ Department of Surgical Oncology, Jackson Memorial Hospital, Miami, FL, USA \\ ${ }^{2}$ Division of Surgical Oncology, DeWitt Daughtry Family Department of Surgery, University of Miami Miller School of Medicine, Miami, FL, USA \\ ${ }^{3}$ Division of Surgical Pathology, University of Miami Miller School of Medicine, Miami, FL, USA
}

\begin{abstract}
Accessory breast tissue is a rare aberration of normal breast development, that presents most commonly in the axilla. Similar to normal breast tissue, it can undergo physiologic and pathologic changes, including malignant transformation. We report a rare case of accessory breast cancer, treated with surgical resection and axillary reverse mapping (ARM), and review current literature focusing on management. We report a 68-year-old female with a history of left breast cancer treated with lumpectomy and axillary dissection, who later developed in-breast recurrence treated with re-lumpectomy and sentinel node biopsy which mapped at the contralateral (right) axilla, but was negative. Two years later screening imaging revealed right axillary tail focal asymmetry with two spiculated masses. Core biopsy showed invasive ductal carcinoma (IDC), and histologic examination of the biopsy could not determine whether this represents a new primary breast cancer or axillary metastasis from the contralateral site. She underwent lumpectomy of the two masses and sentinel node biopsy. During surgery, the masses were identified in the axilla itself, rather than the axillary tail. Final pathology revealed IDC, pT1N0(sn), and extensive ductal carcinoma in situ (DCIS). Due to positive margins, she underwent re-lumpectomy with ARM. Final pathology revealed residual DCIS with negative new margins. The patient was referred for adjuvant radiotherapy. Accessory axillary breast tissue can be confused with axillary tail tissue. It is necessary for the surgeon to distinguish between them by meticulous physical examination and radiologic evaluation, as resection of axillary breast tissue may warrant reverse lymphatic mapping for lymphedema prevention.
\end{abstract}

Keywords: Accessory breast tissue, breast cancer, axillary reverse lymphatic mapping

Cite this article as: Friedman-Eldar O, Melnikau S, Tjendra Y, Avisar E. Axillary Reverse Lymphatic Mapping in the Treatment of Axillary Accessory Breast Cancer: A Case Report and Review of Management. Eur J Breast Health 2022; 18(1): 1-5

\section{Key Points}

- Accessory breast tissue cancer is extremely rare and associated with a worse outcome due to late diagnosis.

- Early diagnosis should be pursued as it can affect management, including the extent of axillary surgery, and the technique for axillary staging.

- Radiation field recommendations in these patients is a data-free zone.

- Reverse lymphatic mapping should be considered as part of the surgical treatment to prevent lymphedema.

\section{Introduction}

Accessory breast tissue $(\mathrm{ABT})$ is a congenital condition in which mammary gland tissue is found outside of the breast. The incidence of ABT is reported to be between $0.3 \%-6 \%$ of females but slightly higher in Asians and Caucasians (1-3). ABT can occur anywhere along the milk line, from axilla to groin, and is thought to result from failure of tissue involution during embryogenesis (4).

ABT can undergo the same physiologic and pathologic changes as normal breast tissue, such as hormonal-induced swelling, inflammatory process, lactational changes, and malignant transformation. Breast cancer originating in ABT represents less than $1 \%$ of all breast cancer cases $(5,6)$. Given its rarity, most of the literature concerning ABT cancer consists of case reports and few case series (7), the largest with 94 patients (8). A second primary invasive carcinoma arising in the contralateral ABT is extremely rare and was described in only one case report previously (7).

Given its location, ABT is not always included in routine screening mammograms, which may lead to delay in diagnosis when cancer develops in it. In addition, due to the proximity of ABT to axillary lymph nodes, ABT cancers have a higher rate of nodal positivity at diagnosis (6), further 
contributing to the worse prognosis in these patients $(1,8-9)$. The majority of cases reported in the literature underwent axillary lymph node dissection (ALND) as part of their surgical treatment (7).

The management of ABT cancer follows the guidelines for pectoral breast cancer and is largely based on the tumor subtype and stage (10). However, for clinically node negative patients that are not undergoing ALND, the rate of post-surgery lymphedema is unknown, and whether the surgical approach should be attenuated to prevent lymphedema has never been evaluated.

We present a case of node negative ABT cancer that was treated surgically by resection concurrent with a lymphedema-preventative approach, axillary reverse mapping (ARM).

\section{Case Presentation}

\section{Clinical course}

A 68-year-old female presented with a history of left breast cancer diagnosed 21 years prior to the current presentation. At the original presentation she was treated with lumpectomy and axillary dissection followed by chemoradiation and 10 years of endocrine therapy. She subsequently presented 19 years later with a recurrence in the left breast that was treated with a second lumpectomy, sentinel node biopsy and intraoperative radiation therapy. The sentinel node mapped in the contralateral (right) axilla as well as in the left internal mammary chain, and all lymph nodes were negative. Two years later, after resumption of aromatase inhibitor treatment, a routine diagnostic mammogram revealed a right axillary tail focal asymmetry with two spiculated masses (Figure 1). Ultrasound confirmed two hypoechoic solid masses in the right axilla, measuring $0.8 \times 0.8 \times 0.4 \mathrm{~cm}$ and $0.7 \times 0.6 \times 0.4 \mathrm{~cm}$ (Figure 2). The patient was asymptomatic but on physical exam a $1 \times 1 \mathrm{~cm}$ axillary tail mass was palpated close to the previous sentinel scar. There was no obvious excess of tissue in the axillary region bilaterally. The rest of her physical exam was unremarkable, except for a well-healed left axillary scar, as well as left lumpectomy and internal mammary lymph node biopsy scars. Ultrasound guided core needle biopsy of one of the masses in the right axilla showed moderately differentiated invasive ductal carcinoma, immunoreactive for estrogen receptor (ER) and progesterone receptor (PR), and not immunoreactive for human epidermal growth factor-2 (HER2). Histologic sections of

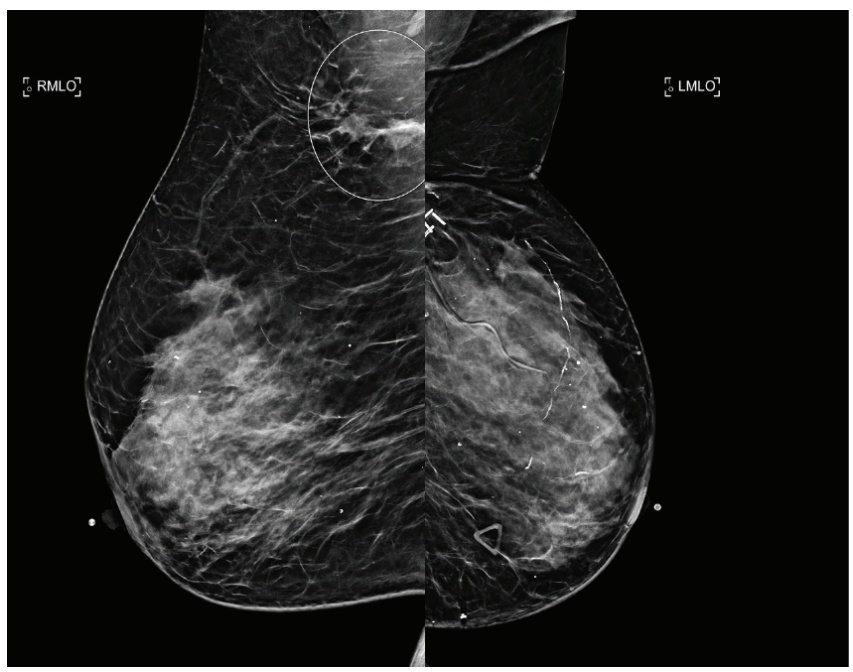

Figure 1. Bilateral mediolateral-view mammography showing right axillary asymmetry (circled) the biopsy specimen showed no definitive evidence of lymph node and/or mammary parenchyma. Therefore, it could not be determined whether this represented a primary mammary carcinoma in the axilla or a lymph node metastasis.

\section{Workup}

Breast magnetic resonance imaging (MRI) was performed and revealed two spiculated homogeneously enhancing masses in the right axillary region measuring $1.6 \mathrm{~cm}$ (with biopsy clip in it) and $1.4 \mathrm{~cm}$. In addition, at least eight morphologically abnormal, level 1, right axillary lymph nodes were identified, the largest measuring up to $1.6 \mathrm{~cm}$ (Figure 3). Ultrasound guided core biopsy was performed on this node with no evidence of malignancy, confirming reactive nodes secondary to coronavirus disease-2019 (COVID-19) vaccine received recently.

Metastatic workup included brain MRI, chest and abdominal computed tomography (CT) scan and bone scan; all were negative.

\section{Treatment}

The case was presented to the multidisciplinary tumor board and since it could not be determined if this was a primary breast cancer or axillary metastasis, the decision was made to proceed with surgical excision of the two masses with sentinel node biopsy, rather than a full axillary dissection.

During surgery, the masses were clearly identified in the axilla itself, rather than the axillary tail. Five "hot" lymph nodes were identified and removed. Histologic examination of the excision specimen revealed moderately differentiated invasive ductal carcinoma (Nottingham Grade 2), $1.6 \mathrm{~cm}$ in greatest dimension with associated extensive intermediate nuclear grade ductal carcinoma in situ (DCIS; Figure 4). Benign mammary parenchyma with dense stroma was identified in the periphery of the lesion. All sentinel lymph nodes were negative for metastatic disease. The overall findings were consistent with carcinoma arising in accessory breast tissue and the final pathologic staging was $\mathrm{pT} 1 \mathrm{~N} 0(\mathrm{sn})$. The posterior margin was focally involved with invasive carcinoma and extensively involved with DCIS, which was also located less than $0.1 \mathrm{~cm}$ from the anterior, superior, and lateral resection margins. Therefore, the patient was taken back to surgery

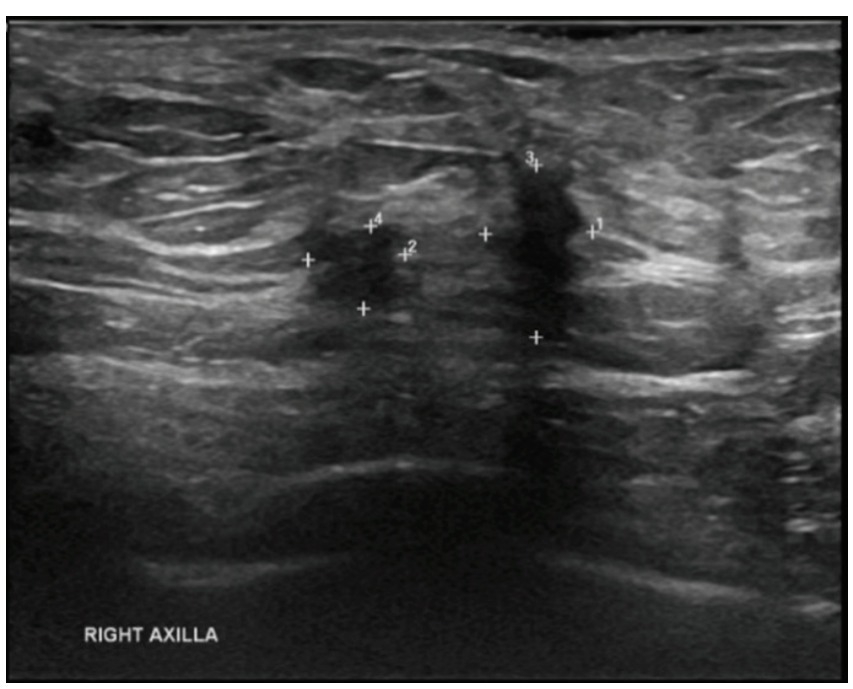

Figure 2. Ultrasonography showing two, ill-defined, hypoechoic, solid masses 
for additional margin resection. Since it was clear that this would entail an extensive axillary manipulation, it was decided to add reverse lymphatic mapping to the procedure, in order to identify and protect arm lymphatics, and possibly lower the risk of lymphedema.

\section{Follow-up}

The patient was seen three weeks after the second surgery. Final pathology revealed residual DCIS with negative new margins. She healed well and was referred for adjuvant radiotherapy, as well as continued endocrine treatment. Genomic profiling with Oncotype Dx was performed on the surgical specimen and revealed a low recurrence score. Therefore, adjuvant chemotherapy was not recommended. She has no evidence of lymphedema, for which surveillance and monitoring is planned.

\section{Discussion and Conclusion}

Ectopic or accessory mammary tissue is most commonly located in the axilla, and development of cancer in it is extremely rare $(4,5)$. This entity presents unique challenges to breast care providers, ranging from surveillance, through diagnosis to management.

Given the atypical location, escape from screening imaging may occur, as well as low level of clinical suspicion on physical exam, often leading to a delay in diagnosis, a more advanced stage at diagnosis, and eventually worsened outcome for ABT cancer patients (8-9). In
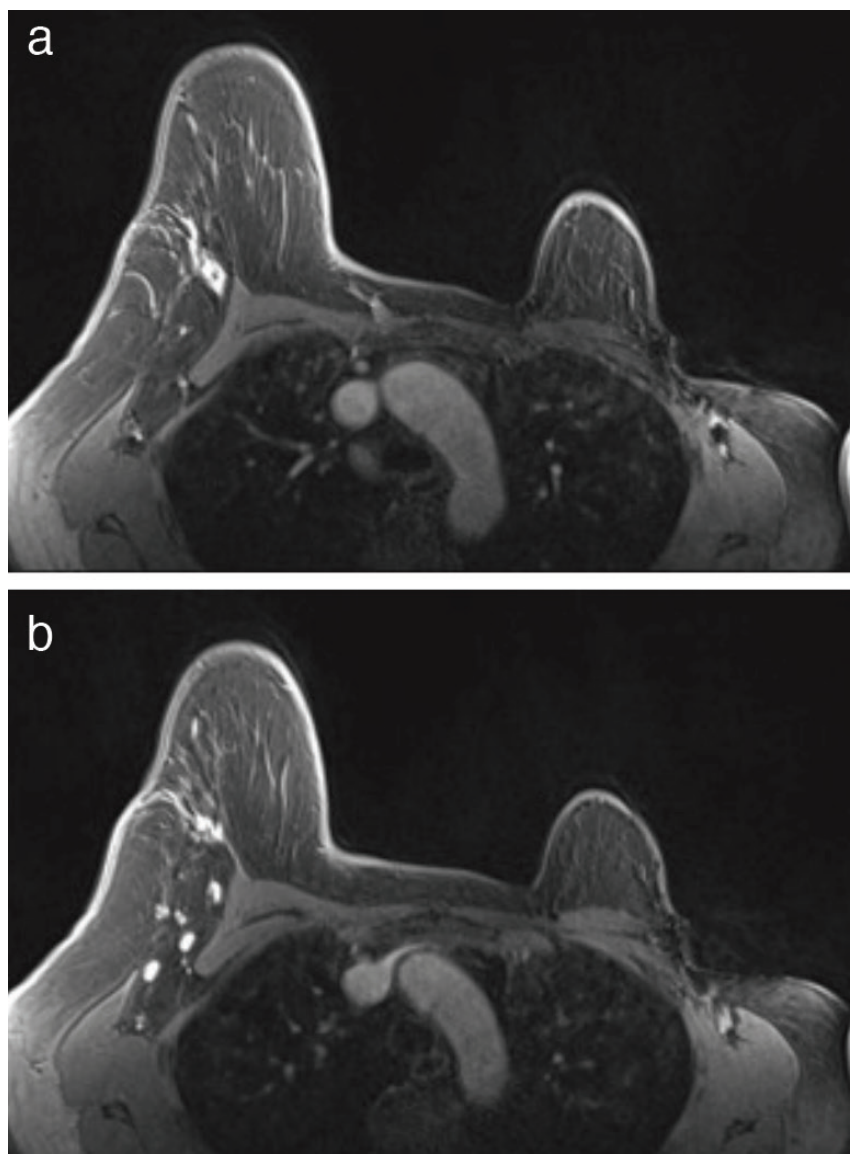

Figure 3. Breast MRI with contrast showing (a) right axillary enhancing mass with a biopsy marker, and (b) several morphologically abnormal right axillary lymph nodes

MRI: Magnetic resonance imaging addition, due to the relatively small amount of breast tissue in the axilla, direct invasion of carcinoma cells to the skin or underlying axillary fibroadipose tissue is more common (7). Axillary nodal involvement may also occur earlier, due to proximity of cancer cells to axillary nodes (6). These particular issues should be taken into account in the assessment of patients with $\mathrm{ABT}$, and especially on the rare occasions when cancer developed in ABT.
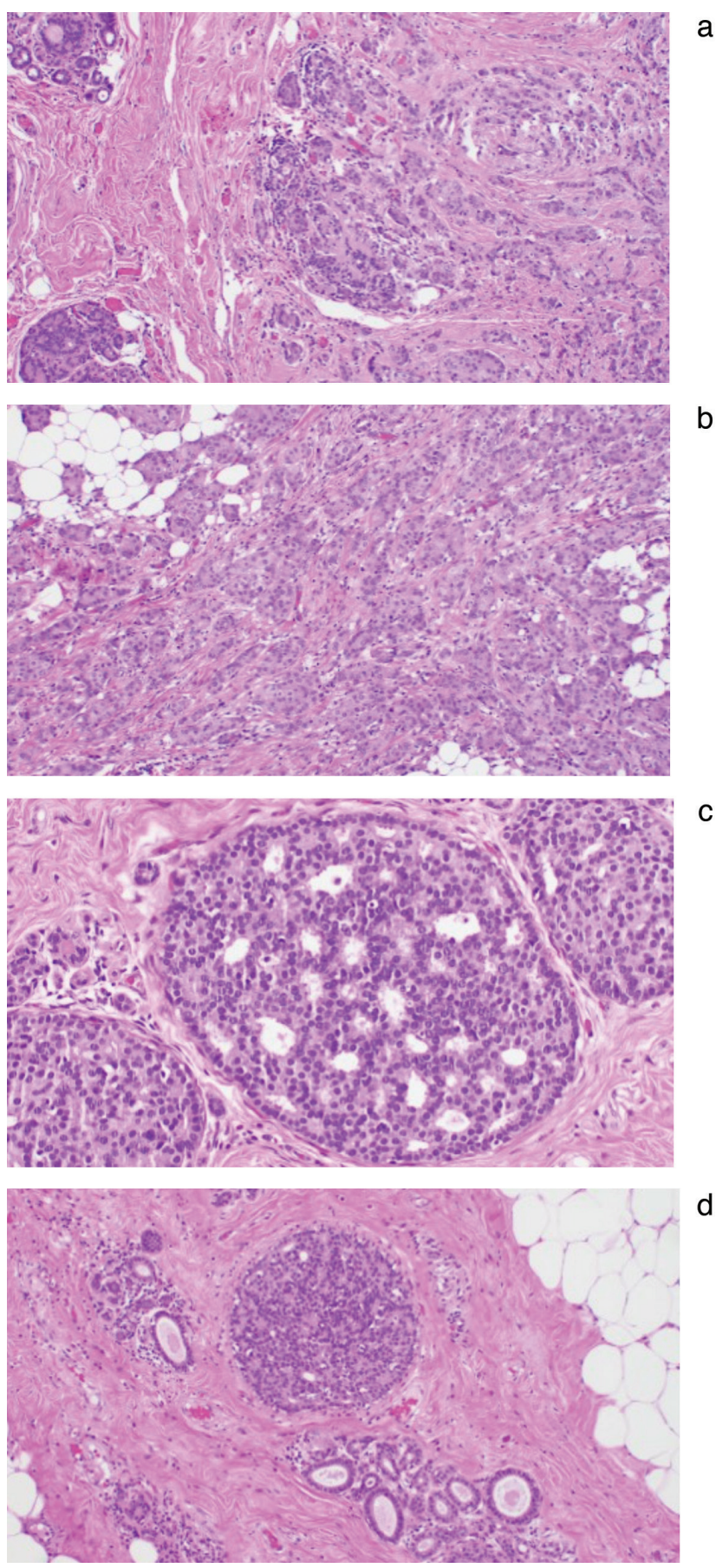

Figure 4. Histologic sections demonstrate moderately differentiated invasive ductal carcinoma (Nottingham Grade 2) [(a-b), Hematoxylin and Eosin (H\&E)]. The invasive carcinoma is associated with ductal carcinoma in situ (DCIS). High power view of intermediate nuclear grade DCIS with cribriform architectural pattern (c). Benign mammary parenchyma with dense intervening stroma is seen in the periphery of the lesion (d) 
As previously stated, the management of $\mathrm{ABT}$ cancers follows the same principles as pectoral breast cancer guidelines (11) and is largely based on tumor subtype and stage (10-11). Still, there are several issues that are essentially different from pectoral breast cancer and require a unique approach. We chose to focus our literature review on those particular issues.

\section{Axillary staging for $\mathrm{cN}$ - patients}

Axillary management can be more challenging in ABT cancer patients due to the close proximity of primary tumor to axillary lymph nodes. In some cases, lymph nodes can create a large conglomerate with the mammary cancer tissue, or can be completely replaced by tumor cells $(12,13)$. Zhang et al. (9) published a case series of 11 patients with ABT cancer and found that more than $80 \%$ of the patients presented with stage 2 or higher disease, and $45 \%$ of them demonstrated axillary nodal disease. According to Maki et al. (14), ALND is the most common surgical approach and is performed in 64\% of cases. Most surgeons advocate routine ALND as part of the primary surgery, due to the higher rate of nodal involvement and the potential for obscuration of sentinel nodes after tracer injection, given their proximity to the tumor (14-17). However, there are reported cases of negative lymph nodes after ALND $(16,18)$, which raises the concern of overtreatment in these patients. Performing sentinel lymph node biopsy (SLNB) for axillary staging in clinically node negative ABT cancer patients continues to be controversial due to the rarity of cases, insufficient literature, and absence of specific management guidelines $(15,19)$. There are case reports of successful SLNB for ABT cancers (7, 19-21), but the reliability of localization and the recommended technique to use are largely unknown. Several case reports have shown successful localization using either radioactive tracer, blue dye or both $(7,21)$. Whether the tracer injection should be located peritumorally in the axilla or in the periareolar region in the breast is another question with no clear answer. Since the embryologic development of ABT occurs independently from that of the breast, it is likely that its lymphatic drainage follows that of the normal anatomy of the armpit, which is towards the ipsilateral axillary nodes and then towards the supraclavicular nodes (2). Therefore it seems more accurate to inject the tracer in the axilla. In several studies, injection was performed peritumorally with good sentinel lymph node identification (21). Preoperative lymphoscintigraphy can sometimes be accompanied with single photon emission computerized tomography (SPECT) for better localization of involved nodes (22).

\section{Adjuvant radiation therapy}

Does the adjacent pectoral breast need to be radiated or only the accessory breast? Furthermore, if we indeed consider ABT as a separate organ from the pectoral breast, can radiation be omitted completely since a "mastectomy" of the whole accessory breast is typically performed, thereby lowering the risk of radiation induced lymphedema? Some authors $(11,23)$ have suggested radiotherapy to the tumor site is indicated if ALND has been avoided to enable local control. However, if sentinel nodes are reliable and negative there should not be a need for this modality, as long as the entire ABT was excised. Those same authors agree that whole breast radiotherapy after surgery is controversial and was not systematically performed. In our opinion, radiation is indicated for partial resection of the $\mathrm{ABT}$ and when a sentinel node cannot be localized while no clinically suspicious disease is present in the axillary nodes. In other cases, the role of adjuvant radiation remains controversial and decided by multidisciplinary teams

\section{Lymphedema rate and prevention}

It is unclear how the resection of ABT cancer itself affects the rate of lymphedema, even when ALND is not performed. Factors such as size of excised tissue and number of sentinel nodes removed are important, as well as adjuvant radiation to the axilla (24). Several techniques have been developed to minimize the risk of arm lymphedema among patients undergoing axillary surgery. One of them is the axillary reverse lymphatic mapping (ARM) technique, in which a tracer is injected into the ipsilateral upper extremity, allowing the surgeon to visualize and preserve lymphatic channels and lymph nodes draining the arm, thereby minimizing disruption of lymphatic flow (25). Several studies have reported decreased lymphedema rate when ARM was performed along with ALND (26-28) or even only SLNB (29-30). In the case of ABT cancer surgery it seems reasonable to consider ARM routinely, given the axillary manipulation performed, even if SLNB alone is used. In the event that ALND is necessary, ARM may have a higher impact, and can also serve as the first step to a Lymphatic Microsurgical Preventive Healing Approach (LYMPHA) (31) or S-LYMPHA (Simplified LYMPHA) (32). To the best of our knowledge, this is the first case report describing ARM performance in association with ABT cancer surgery.

In the case presented there was no clinically apparent swelling in the axillae to suggest the presence of ABT, most probably due to the patient's post-menopausal state and her previous axillary surgeries bilaterally (left axillary dissection and right sentinel node biopsy). In retrospect, further anamnesis taken after the right lumpectomy and SLNB revealed episodes of cyclical axillary swelling during her fertility years. The swelling was especially noticeable to her during pregnancy and post-partum.

Learning from this experience, we recommend clinicians to get a full ABT-directed anamnesis for every patient with axillary tail cancer. In addition, raising the clinical suspicion in multi-disciplinary meetings can further assist with meticulous radiologic evaluation of the anatomical localization of the tumor, as well as discussing treatment options that are unique to this condition, especially in relation to extent of surgery, injection site for sentinel node biopsy, adjuvant radiation and lymphedema prevention. Breast care providers must be aware of this entity, and the unique challenges it poses.

In conclusion, cancer arising in axillary $\mathrm{ABT}$ is extremely rare and associated with worse outcome due to late diagnosis. It is important to diagnose cancer in ABT before surgery, as it can affect management decisions, such as the extent of axillary surgery, technique of axillary staging, radiation recommendations and reverse lymphatic mapping to prevent lymphedema. Awareness and a high level of suspicion are key.

Ethics Committee Approval: The case report has been exempted by the ethical committee of our institution (EXMPT-26-12-18-01).

Informed Consent: Written informed consent was obtained from the patient for publication of this case report and accompanying images.

Peer-review: Externally peer-reviewed.

\section{Authorship Contributions}

Surgical and Medical Practices: O.F.E., S.M., Y.T., E.A.; Concept: O.F.E., S.M., E.A.; Design: O.F.E., E.A.; Data Collection and/or Processing: O.F.E., S.M., 
Y.T., E.A.; Analysis and/or Interpretation: O.F.E., S.M., Y.T., E.A.; Literature Searching: O.F.E., S.M.; Writing: O.F.E., S.M., Y.T., E.A.

Conflict of Interest: No conflict of interest declared by the authors.

Financial Disclosure: The authors declare that this study received no financial disclosure.

\section{References}

1. Gutermuth J, Audring H, Voit C, Haas N. Primary carcinoma of ectopic axillary breast tissue. J Eur Acad Dermatol Venereol 2006; 20: 217-221. (PMID: 16441639) [CrossRef]

2. Schmidt H. Supernumerary nipples: prevalence, size, sex and side predilection - a prospective clinical study. Eur J Pediatr 1998; 157: 821823. (PMID: 9809822) [CrossRef]

3. Lim SY, Jee SL, Gee T, Nor Aina E. Axillary accessory breast carcinoma masquerading as axillary abscess: a case report. Med J Malaysia 2016; 71 : 370-371. (PMID: 28087969) [CrossRef]

4. DeFilippis EM, Arleo EK. The ABCs of accessory breast tissue: basic information every radiologist should know. AJR Am J Roentgenol 2014; 202: 1157-1162. (PMID: 24758674) [CrossRef]

5. Giron GL, Friedman I, Feldman S. Lobular carcinoma in ectopic axillary breast tissue. Am Surg 2004; 70: 312-315. (PMID: 15098783) [CrossRef]

6. Francone E, Nathan MJ, Murelli F, Bruno MS, Traverso E, Friedman D. Ectopic breast cancer: case report and review of the literature. Aesthetic Plast Surg 2013; 37: 746-749. (PMID: 23620009) [CrossRef]

7. Addae JK, Genuit T, Colletta J, Schilling K. Case of second primary breast cancer in ectopic breast tissue and review of the literature. BMJ Case Rep 2021; 14: e241361. (PMID: 33832937) [CrossRef]

8. Nihon-Yanagi Y, Ueda T, Kameda N, Okazumi S. A case of ectopic breast cancer with a literature review. Surg Oncol 2011; 20: 35-42. (PMID: 19853438) [CrossRef]

9. Zhang S, Yu YH, Qu W, Zhang Y, Li J. Diagnosis and treatment of accessory breast cancer in 11 patients. Oncol Lett 2015; 10: 1783-1788. (PMID: 26622750) [CrossRef]

10. Famá F, Cicciú M, Sindoni A, Scarfó P, Pollicino A, Giacobbe G, et al. Prevalence of Ectopic Breast Tissue and Tumor: A 20-Year Single Center Experience. Clin Breast Cancer 2016; 16: e107-e112. (PMID: 27117240) [CrossRef]

11. National Comprehensive Cancer Network. NCCN clinical practice guidelines in oncology (NCCN guidelines ${ }^{\circ}$ ). Breast Cancer 2020 Last Accessed Date: 01.07.2021. Available at: https://www. nccn. org/ professionals/ physician_ gls/pdf/ breast.pdf. [CrossRef]

12. Bi M, Li D, Su Y, Sun P, Gao Y. Male axillary accessory breast cancer: A case report. Medicine (Baltimore) 2020; 99: e19506. (PMID: 32176095) [CrossRef]

13. Pardo M, Silva F, Jiménez P, Karmelic M. [Mammary carcinoma ine ectopic breast tissue. A case report.] Rev Med Chil 2001; 129: 663-665. (Article in Spanish) (PMID: 11510208) [CrossRef]

14. Maki T, Murakami A, Morishita M, et al. Right axillary accessory breast cancer. Rinsho Derma 2015;57:1147-1151. [CrossRef]

15. Zhang J, Zhang W, Min M, Pan Y. Axillary accessory breast cancer with persistent left superior vena cava: A case report and treatment controversy. Int J Surg Case Rep 2020; 73: 71-74. (PMID: 32645595) [CrossRef]

16. Wang CX, Guo SL, Han LN. Successful treatment of accessory breast cancer with endocrine therapy. J Zhejiang Univ Sci B 2017; 18: 70-75. (PMID: 28070998) [CrossRef]

17. Hao JY, Yang CC, Liu FF, Yang YL, Li S, Li WD, et al. Accessory breast cancer occurring concurrently with bilateral primary invasive breast carcinomas: a report of two cases and literature review. Cancer Biol Med 2012; 9: 197-201. (PMID: 23691479) [CrossRef]

18. Takahashi E, Terata K, Nanjo H, Ishiyama K, Hiroshima Y, Yamaguchi A, et al. A male with primary accessory breast carcinoma in an axilla is strongly suspected of having hereditary breast cancer. Int Cancer Conf J 2021; 10: 107-111. (PMID: 33782642) [CrossRef]

19. Khan RN, Parvaiz MA, Khan AI, Loya A. Invasive carcinoma in accessory axillary breast tissue: A case report. Int J Surg Case Rep 2019; 59: 152155. (PMID: 31163330) [CrossRef]

20. Bröker ME, Bekken JA, Reijnen MM, Bröker WF. [Breast cancer in an accessory breast.] Ned Tijdschr Geneeskd 2011; 155: A3638. (Article in German) (PMID: 22008157) [CrossRef]

21. Patel BK, Jafarian N, Abbott AM, Khazai L, Lee MC. imaging findings and management of primary breast cancer in accessory axillary breast tissue. Clin Breast Cancer 2015; 15: e223-e229. (PMID: 25986957) [CrossRef]

22. Leroy-Freschini B, Loussert L. Unexpected breast lymphoscintigraphy findings after surgical removal of accessory mammary gland. Breast J 2020; 26: 769-770. (PMID: 31544325) [CrossRef]

23. Routiot T, Marchal C, Verhaeghe JL, Depardieu C, Netter E, Weber B, et al. Breast carcinoma located in ectopic breast tissue: a case report and review of the literature. Oncol Rep 1998; 5: 413-417. (PMID: 9468570) [CrossRef

24. DiSipio T, Rye S, Newman B, Hayes S. Incidence of unilateral arm lymphoedema after breast cancer: a systematic review and meta-analysis. Lancet Oncol 2013; 14: 500-515. (PMID: 23540561) [CrossRef]

25. Thompson M, Korourian S, Henry-Tillman R, Adkins L, Mumford S, Westbrook KC, et al. Axillary reverse mapping (ARM): a new concept to identify and enhance lymphatic preservation. Ann Surg Oncol 2007; 14: 1890-1895. (PMID: 17479341) [CrossRef]

26. Beek MA, Gobardhan PD, Klompenhouwer EG, Rutten HJ, Voogd AC, Luiten EJ. Axillary reverse mapping (ARM) in clinically node positive breast cancer patients. Eur J Surg Oncol 2015; 41: 59-63. (PMID: 25468747) [CrossRef]

27. Nos C, Clough KB, Bonnier P, Lasry S, Le Bouedec G, Flipo B, et al. Upper outer boundaries of the axillary dissection. Result of the SENTIBRAS protocol: Multicentric protocol using axillary reverse mapping in breast cancer patients requiring axillary dissection. Eur J Surg Oncol 2016; 42: 1827-1833. (PMID: 27769634) [CrossRef]

28. Yuan Q, Wu G, Xiao SY, Hou J, Ren Y, Wang H, et al. Identification and Preservation of Arm Lymphatic System in Axillary Dissection for Breast Cancer to Reduce Arm Lymphedema Events: A Randomized Clinical Trial. Ann Surg Oncol 2019; 26: 3446-3454. (PMID: 31240591) [CrossRef]

29. Abdelhamid MI, Bari AA, Farid MI, Nour H. Evaluation of axillary reverse mapping (ARM) in clinically axillary node negative breast cancer patients - Randomised controlled trial. Int J Surg 2020; 75: 174-178. (PMID: 32059974) [CrossRef]

30. Faisal M, Sayed MG, Antonious K, Abo Bakr A, Farag SH. Prevention of lymphedema via axillary reverse mapping for arm lymph-node preservation following breast cancer surgery: a randomized controlled trial. Patient Saf Surg 2019; 13: 35. (PMID: 31807140) [CrossRef]

31. Boccardo F, Casabona F, De Cian F, Friedman D, Murelli F, Puglisi M, et al. Lymphatic microsurgical preventing healing approach (LYMPHA) for primary surgical prevention of breast cancer-related lymphedema: over 4 years follow-up. Microsurgery 2014; 34: 421-424. (PMID: 24677148) [CrossRef]

32. Tolga Ozmen, Mesa Lazaro, Yan Zhou, Alicia Vinyard, Eli Avisar Evaluation of Simplified Lymphatic Microsurgical Preventing Healing Approach (S-LYMPHA) for the Prevention of Breast Cancer-Related Clinical Lymphedema After Axillary Lymph Node Dissection. Ann Surg 2019; 270: 1156-1160. (PMID: 29794843) [CrossRef] 